\title{
Der Einfluss von gereinigtem Abwasser auf das Phytoplankton in Seen
}

\author{
Von P. Bossard und H. AmbüHL \\ Eidg. Technische Hochschule Zürich \\ Institut für Gewässerschutz und Wassertechnologie ${ }^{1}$ ) \\ Manuskript eingegangen am 26. Mai 1974
}

\section{ABSTRACT}

\section{Effect of treated sewage effluents on phytoplankton in lakes}

The effect of domestic sewage effluents after a) mechanical, b) mechanical-biological and c) mechanical-biological-chemical treatment on algal cultures and on natural lake phytoplankton was studied by means of laboratory assays and in situ experiments. The concentrations of phosphorus and nitrogen are almost exclusively responsible for any algal growth. Other constituents which may be present in sewage effuents (e.g. residual amounts of iron originating from the tertiary treatment) do not stimulate substantially the growth of algac.

\section{Einleitung}

Die heute gebräuchlichen Verfahren der Abwasserreinigung, insbesondere die Belebtschlammbehandlung und die chemische Fällung, ermöglichen Nährstoffkonzentrationen im Endabfluss der Kläranlage von weniger als $1 \mathrm{mg} / \mathrm{l}$ Phosphor und etwa 10-15 mg/l Stickstoff. Bei Einleitung in die photische Schicht eines Sees können derartige Konzentrationen, besonders jene des Phosphors, immer noch eine Steigerung der Photosynthese hervorrufen. Auch wenn das Abwasser beim Eintritt in den See verdünnt wird, kann die lokale Beeinflussung unerwünschte Ausmasse annehmen. GÄCHTER et al. [1] wiesen im mesotrophen Vierwaldstättersee Eutrophierungserscheinungen in der zwei Quadratkilometer grossen Horwer Bucht nach. Die Ursache daftir bildete eindeutig ein mit Abwasser belasteter Bach, dessen Phosphorkonzentration einige $\mathrm{mg} / \mathrm{l}$ betrug. Der in den Richtlinien über die Beschaffenheit abzuleitender $A b$ wässer vorgeschriebene Grenzwert von $0,5 \mathrm{mg} / \mathrm{l} \mathrm{P}$ ist mit der heutigen Reinigungstechnik kaum noch zu unterbieten. Bei optimaler Ausnützung würde diese P-Kon-

1) Die Arbeit wurde an der Eidg. Anstalt für Wasserversorgung, Abwasserreinigung und Gewässerschutz (EAWAG) ausgeführt. 
zentration die Synthese von $500 \mathrm{~g}$ Algen-Naßsubstanz pro $\mathrm{m}^{3}$ eingeleiteten Abwassers ermöglichen, sofern mit reinen Nährstoffen gerechnet wird und weitere Stimulantien ausser acht gelassen werden.

In den bis heute bekanntgewordenen Studien über die Düngewirkung von Pflanzennährstoffen auf planktische Organismen wurde mit wenigen Ausnahmen mit reinen Salzen gearbeitet. Nun ist in dem von uns anvisierten, sehr häufigen und praktisch bedeutsamen Fall zu beachten, dass in gereinigtem Abwasser neben dem relativ geringen Anteil anorganischer Dünger organische Stoffe in verhältnismässig grosser Menge vorliegen, darunter solche, die unmittelbar in das stoffliche Geschehen eingreifen können (natürliche und künstliche Komplexbildner im besonderen), ferner Stoffe, welche in geringsten Mengen wachstumsstimulierend oder auch toxisch wirken können ( $\mathrm{Cu}, \mathrm{Mo}, \mathrm{Co}, \mathrm{Fe}$ ), je nachdem, in welcher Form sie vorliegen und welche Kondition das gesamte stoffliche System im Augenblick besitzt. Bei gereinigtem Siedlungsabwasser sind in dieser Hinsicht Eisen (als unvermeidlich im Abwasser verbleibende Reste des Fällungsmittels der «dritten Reinigungsstufe») und nicht abgebaute komplexierende Waschmittelanteile besonders zu beachten, daneben aber auch verdünnte Abgänge aus metallverarbeitenden und -veredelnden Betrieben. Eutrophierungsexperimente an Fliesswassersystemen haben gezeigt, dass eine Düngewirkung durch reine Nährsalze $(\mathrm{P}+\mathrm{N})$ erst dann in grösserem Umfang auftritt, wenn kleinste Mengen von häuslichem Abwasser (0,1-0,5 Vol.-\%) zugegeben werden [2]. Vergleichbare Kenntnisse aus stehenden Gewässern fehlen weitgehend; sie sind aber für die Bewertung der finalen ökologischen Wirkungsleistung der heutigen Klärsysteme erforderlich.

Pauschal haben die bisherigen Arbeiten folgendes ergeben: Phosphor und Stickstoff sind die wirksamsten und gegenüber allen andern Nährstoffen mit grossem Abstand wichtigsten produktionslimitierenden Komponenten. Komplexbildner und Schwermetalle (Spurenelemente) können die Produktion beeinflussen, doch ist dieser Einfluss verhältnismässig klein. Diese Feststellungen wurden an Seen aller Trophiestufen in Europa und Amerika gemacht und dürften daher als allgemeingültig betrachtet werden. Bezeichnend ist aber, dass sie sich weitgehend auf reine Testsubstanzen beziehen; die produktionsbiologische Wirkung von Abwasser wurde dagegen bisher nur in vereinzelten Arbeiten geprüft. Wir versuchen im folgenden, zusammen mit den Resultaten einer eigenen experimentellen Untersuchung, diese Arbeiten auszuwerten.

\section{Generelle Problemstellung}

$\mathrm{Zu}$ prüfen ist die Reaktion des natürlichen Phytoplanktons auf die Zugabe von verschiedenen behandelten Abwässern sowie vergleichsweise von reinen Nährstoffen:

1. Die Wachstumsreaktionen des Phytoplanktons auf die Zugabe gleicher Mengen von rohem, mechanisch, mechanisch-biologisch und mechanisch-biologisch-chemisch gereinigtem Abwasser sollen untereinander verglichen werden. Falls die verschieden behandelten Abwässer verschiedenartige ökologische Effekte auslösen, stellt sich die Frage, welche einzelnen Faktoren dafür verantwortlich sind.

2. Die Wachstumsreaktionen des Phytoplanktons auf die Zugabe von gereinigtem Abwasser und äquivalenten Mengen von reinen $\mathrm{P}$ - und N-Salzen sollen untereinander verglichen werden. 


\section{Experimentelle Untersuchungen}

Im folgenden wird versucht, aus den Resultaten einer eigenen Untersuchung und einschlägigen publizierten Arbeiten eine Synthese zu erarbeiten, welche mindestens die Fragen der Praxis beantwortet. Keine dieser Arbeiten gibt über sämtliche Fragen der Problemstellung Aufschluss. Ferner sind die angewandten Methoden und Objekte sehr verschieden, was in gewisser Hinsicht einer summarischen Beantwortung der angeführten Fragen dienlich ist, direkte Vergleiche von Experimenten jedoch erschwert. Eine gründliche Abklärung der Probleme mit einer einheitlichen Methodik würde weitere unbestimmte Zeit in Anspruch nehmen, da im Gang befindliche methodische Arbeiten abzuwarten und weitere methodische Fragen überhaupt erst noch zu klären wären. Wir glauben deshalb, mit einer Interpretation der heute vorhandenen Kenntnisse der Sache besser dienen zu können.

\subsection{Eigene Arbeiten}

Der Einfluss von zwei- und dreistufig $\left.{ }^{1}\right)$ gereinigtem Siedlungsabwasser wurde mit Labortests (Bioassays) und In-situ-Experimenten im mesotrophen Vierwaldstättersee untersucht.

\section{Bioassays}

Batchkulturen von 21 Inhalt wurden mit $0,2 \mu$ filtriertem Vierwaldstätterseewasser (VSW) angesetzt, mit der Grünalge Scenedesmus obliquns geimpft und mit gereinigten Abwässern und reinen Nährstoffen bei Versuchsanfang gedüngt. Die Düngung erfolgte mit $0,2 \mu$ filtriertem zwei- und dreistufig gereinigtem Abwasser, mit $\mathrm{NaNO}_{3}$ sowie $\mathrm{KH}_{2} \mathrm{PO}_{4}$ (autoklaviert). Nach dem Abfüllen der Nährlösungen und Algensuspensionen wurden die Kulturflaschen einer konstanten Lichtquelle (6000 Lux) ausgesetzt und an ein Pressluft- $\mathrm{CO}_{2}$-Gemisch angeschlossen. Die Biomassebestimmung erfolgte durch tägliches Auszählen der Algenzellen unter dem Mikroskop in der Bürker-Kammer. Nach sieben Tagen Versuchsdauer wurden der Gesamtphosphor roh und filtriert $(0,45 \mu$ Millipore), Phosphat, Nitrat und Nitrit untersucht. Das Experiment wurde im Juli 1972 ausgeführt und im November wiederholt. Im folgenden wird das November-Experiment detaillierter geschildert (Abb. 2). Das JuliExperiment zeigte qualitativ die gleichen Resultate (Abb. 1).

Versuchsanordnung

\begin{tabular}{lccc}
\hline & \multicolumn{2}{c}{ Anfangskonzentrationen } \\
& $\mu g \mathrm{PO}_{\mathbf{4}}-\mathrm{P} / \mathrm{l}$ & $\mu \mathrm{g}$ Ges.-P/l & $\mu^{2} \mathrm{NO}_{\mathbf{3}}-\mathrm{N} / \mathrm{I}$ \\
\hline Kontrolle : Vierwaldstätterseewasser (VSW) & 7 & 23 & 360 \\
VSW + dreistufig gereinigtes Abwasser & 21 & 36 & 540 \\
VSW + zweistufig gereinigtes Abwasser & 80 & 103 & 500 \\
VSW + Phosphat + Nitrat & 94 & 99 & 480 \\
VSW + dreistufig gereinigtes Abwasser + Phosphat & 93 & 100 & 500 \\
\hline
\end{tabular}

Anfangskonzentration der Scenedesmus-Zellen: $10^{3}$ Zellen pro $\mathrm{ml}$.

$$
\text { Resultate (Abb. 2) }
$$

Am sechsten Tag wurde in den meisten Kulturflaschen die maximale Zellzahl erreicht. Am siebten Tag begannen die Algen teilweise bereits abzusterben. Bei Versuchsende wurden folgende Werte gemessen: 


\begin{tabular}{|c|c|c|c|}
\hline & $\begin{array}{l}\text { Gelöster P } \\
\mu \mathrm{g} / 1\end{array}$ & $\begin{array}{l}\text { Endkonzentrationen } \\
\mu g / 1 \text { partikulärer } \mathrm{P} \\
\text { am } 7 \text {.'Tag }\end{array}$ & $\begin{array}{l}\text { Zellzahl } / \mathrm{ml} \\
\text { am 6.Tag }\end{array}$ \\
\hline Kontrolle: VSW & 7 & 14 & $1,0 \times 10^{5}$ \\
\hline VSW + dreistufig gereinigtes Abwasser & 7 & 28 & $3,2 \times 10^{5}$ \\
\hline VSW $+z w e i s t u f i g$ gereinigtes Abwasser & 9 & 98 & $7,2 \times 10^{5}$ \\
\hline VSW + Phosphat + Nitrat & 7 & 105 & $7,1 \times 10^{5}$ \\
\hline $\begin{array}{l}\text { VSW + dreistufig gereinigtes Abwasser+ } \\
\text { Phosphat }\end{array}$ & 8 & 102 & $7,2 \times 10^{5}$ \\
\hline
\end{tabular}

1) "Zweistufig gereinigt»: mechanisch-biologisch gereinigtes Abwasser; "dreistufig gereinigt»: mechanisch-biologisch-chemisch gereinigtes Abwasser.
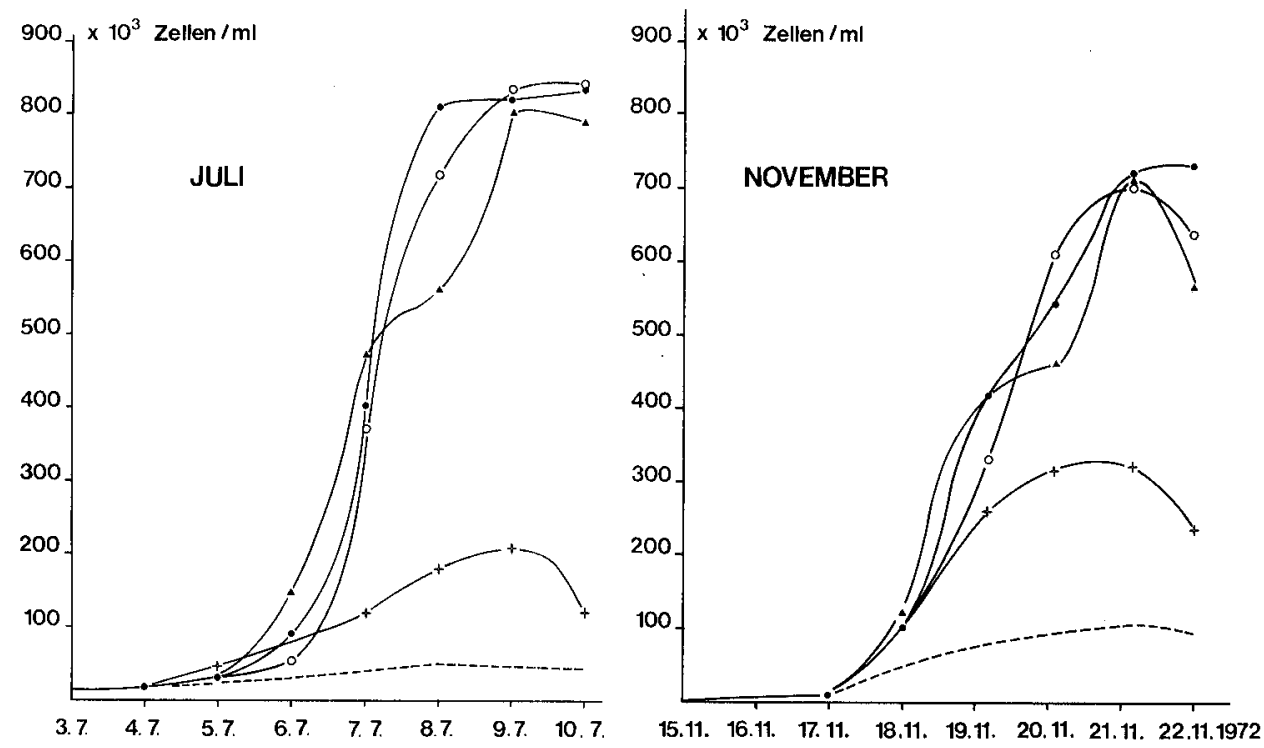

Abb. 1. Bioassay (Labor-Batch-Kultur) mit Scenedesmus obliquus; zeitlicher Verlauf der Zelldichte pro $\mathrm{ml}$ Kulturlösung.

$$
7----- \text { Kontrolle }
$$

$2-2$ 2stufig gereinigtes Abwasser zugesetzt

$3+-+3$ stufig gereinigtes Abwasser zugesetzt

$4 \boldsymbol{\Delta}-\boldsymbol{\Delta}$ 3stufig gereinigtes Abwasser + P-Zusatz

$5 \bigcirc-\bigcirc$ Zusatz reiner Phosphat-Nitrat-Lösung

Versuch vom 3. bis 10. Juli 1972.

Fig. 1. Bioassay (laboratory batch-cultures) with Scenedesmus obliquus; cell density per $\mathrm{ml}$ of culture solution versus time.

$$
1 \text { - - - - - Control }
$$

2 - Two-stage treated sewage added

$3+-+$ Three-stage treated sewage added

$4 \boldsymbol{\Delta} \Delta$ Three-stage treated sewage + P-addition

$50-O$ Addition of pure phosphate-nitrate solution

Test period: 3-10 July 1972.

Abb. 2. Bioassay mit Scenedesmus obliquus; Anordnung wie in Abb. 1. Versuch vom 15. bis 22. November 1972.

Fig. 2. Bioassay with Scenedesmus obliquus; same symbols as in Figure 1. Test period: 15-22 November 1972. 
Das zweistufig gereinigte Abwasser, das mit Phosphat aufgestockte dreistufig gereinigte Abwasser und die kombinierte Phosphat-Nitrat-Düngung bewirkten gegenüber der Kontrolle eine Versiebenfachung der Zellzahl nach sieben Tagen. Das dreistufig gereinigte Abwasser verdreifachte die Zellzahl gegenüber der Kontrolle.

\section{Folgerungen}

Sowohl zwei- als auch dreistufig gereinigtes Abwasser zeigte einen Düngeeffekt im Vergleich zur Kontrolle. Reines Phosphat und Nitrat zeigten dieselbe Düngewirkung wie zweistufig gereinigtes Abwasser mit gleichem Phosphorgehalt. Der Unterschied der Düngewirkung von zwei- und dreistufig gereinigtem Abwasser auf das Wachstum von Scenedesmus obliquus in Kultur ist demnach einzig auf den unterschiedlichen Phosphatgehalt zurückzuführen.

\section{In-situ-Versuche}

Es wurde die Methode von BürGI [3] übernommen: Testzellen von 60001 Inhalt umschliessen im See eine Wassersäule von $10 \mathrm{~m}$ Höhe. Die Versuchsanordnung war mit derjenigen der Laborversuche identisch. Als Testbiozönose diente das originale, unveränderte Plankton des Vierwaldstättersees. Im Unterschied zur einmaligen Düngung in den Batchkulturen wurden die Nährstoffe täglich in Raten von etwa $10 \mu \mathrm{g} / \mathrm{P}$ zugedüngt. Vom Mai bis Juli 1972 fanden drei Versuche statt, deren Dauer 7-15 Tage betrug. Aus arbeitstechnischen Gründen musste auf die direkte Biomassebestimmung verzichtet werden. Als Ersatzparameter wurden Chlorophyll und partikulärer Phosphor bestimmt. Mit diesen Versuchen sollten die Resultate der Labor-Algentests unter möglichst wirklichkeitsnahen Bedingungen bestätigt werden.

Versuchsanordnung

Kontrolle: Vierwaldstätterseewasser (VSW)

$\mathrm{VSW}+$ dreistufig gereinigtes Abwasser

VSW + zweistufig gereinigtes Abwasser

VSW + Phosphat + Nitrat (äquivalent zu den betreffenden Fraktionen im zweistufig gereinigten Abwasser)

VSW + dreistufig gereinigtes Abwasser + Phosphat (aufgestockt auf die P-Konzentration von zweistufig gereinigtem Abwasser)

\section{Resultate}

Obwohl durch die semikontinuierliche Düngung insgesamt 20-140 $\mu \mathrm{g} / \mathrm{l}$ Phosphat-P dem Seewasser zugegeben wurden, war in sämtlichen Experimenten im Vergleich zur Kontrolle und im Vergleich zu den respektiven Anfangskonzentrationen kein wesentlicher Anstieg an Chlorophyll und partikulärem Phosphor zu verzeichnen. Während der Experimente waren im See folgende Werte festzustellen:

$\begin{array}{lc}\text { Chlorophyll (bestimmt nach SCOR) } & 60-80 \mathrm{mg} / \mathrm{m}^{2} \\ \text { Gesamtphosphor } & 10-25 \mu \mathrm{g} / \mathrm{l} \\ \text { Partikulärer Phosphor } & 8-20 \mu \mathrm{g} / \mathrm{l} \\ \text { Phosphatphosphor } & 0-4 \mu \mathrm{g} / \mathrm{l} \\ \text { Nitratstickstoff } & 200-300 \mu \mathrm{g} / \mathrm{l}\end{array}$

Es herrschten somit ausgesprochen oligotrophe Verhältnisse.

Während in einem Experiment nach achttägiger Düngung 50-100\% des zugedüngten Phosphors in den Testzellen grösstenteils als Phosphat wieder gefunden 
wurden, verschwand in den beiden andern Experimenten der zugedünte Phosphor aus der Wassersäule mit grösster Wahrscheinlichkeit durch die untere Öffnung. Bezeichnenderweise verhielt sich das zugedüngte Nitrat ähnlich. Das Fehlen eines messbaren Biomassezuwachses, der unzweifelhaft vorhanden sein muss, wenn während der Versuche kein freies Orthophosphat festgestellt werden kann und dieses somit bei der semikontinuierlichen Düngung laufend aufgebraucht worden sein muss, lässt eine vermehrte Sedimentation vermuten, was bei den in den Testzellen gegenüber der Umgebung leicht veränderten Turbulenzverhältnissen plausibel wäre. Das dem Phosphat analoge Verhalten von Nitrat bestärkt diese Vermutung.

Andere Versuche mit Testzellen haben gezeigt, dass dieses Verfahren zwar ein Resultat liefert, welches auf den Biotop unmittelbar anwendbar ist. Es weist aber grosse Streuungen auf und sollte daher trotz dem grossen Aufwand nur mit der statistisch erforderlichen Anzahl von Parallelen angewendet werden.

\section{2 «Shagawa Lake Project» [4]}

Wertvolle Resultate stammen aus Versuchen, welche in den Jahren 1969 ff. zur Abklärung von Sanierungsfragen am Lake Shagawa (Minnesota, USA) ausgeführt wurden. Dieser See hat eine Oberfläche von rund $5 \mathrm{~km}^{2}$ bei einer mittleren Tiefe von etwa $15 \mathrm{~m}$. Sein Hauptzufluss stammt aus einem oligotrophen See; für seinen trotzdem eutrophen Zustand ist allein der Abwasserzufluss aus einer 5000 Einwohner zählenden Gemeinde verantwortlich. Diese Belastung besteht seit 1900. Ab 1954 wurde das Abwasser mechanisch gereinigt; später wurde ein hochbelasteter Tropfkörper angeschlossen. Seit 1973 wird das Abwasser in einer dreistufigen Anlage behandelt. Die hier referierten Experimente aus diesem Projekt sind insofern bemerkenswert, als es sich um Grossversuche handelt, deren Resultate - mit einiger Reserve - unmittelbar auf das Gewässer übertragen werden dürfen.

Malueg et al. [4] prüften die Düngewirkung von zwei- und dreistufig gereinigtem Abwasser auf das Phytoplankton des Lake Shagawa in drei durchsichtigen, je $600 \mathrm{~m}^{3}$ fassenden Bassins. Aus den täglich gemessenen Chlorophyllwerten (als Biomasse-Parameter) ist zu schliessen, dass das zweistufig gereinigte Abwasser das Algenwachstum im allgemeinen stimuliert, während das dreistufig gereinigte gegenüber der Kontrolle keine wesentliche Wirkung zeigt. In Ergänzung dazu prüften PoWERS et al. [5] den Einfluss von zweistufig gereinigtem Abwasser und von reinen Nährstoffen auf das Phytoplankton des Lake Shagawa, und zwar in halbtechnischen In-situ-Experimenten. Als Testgefässe dienten $2 \mathrm{~m}$ lange, senkrecht eingehängte Schläuche mit einem Volumen von rund 320 l, welche mit Wasser aus dem Lake Shagawa oder vergleichsweise mit Wasser aus dem Abfluss des oligotrophen Oberliegersees gefüllt wurden. Das zu prüfende Plankton stammte immer aus dem Lake Shagawa. Folgende Fragestellung bzw. Versuchsanordnung wurde getroffen:

1. Düngewirkung von reinem Phosphat in verschiedenen Konzentrationen; Vergleich mit der Düngewirkung von zweistufig gereinigtem Abwasser.

2. Düngewirkung von reinem Phosphat + Nitrat; Vergleich mit zweistufig gereinigtem Abwasser.

3. Prüfung, ob im Abwasser neben Phosphat und Nitrat weitere wachstumsfördernde Faktoren enthalten sind. 
Resultate: Beide untersuchten Nährstoffe (Phosphat und Nitrat) können die Algenproduktion im Lake Shagawa limitieren. Ist Nitrat in ausreichender Menge vorhanden, so bestimmt Phosphat die Algenbiomasse, gemessen am Chlorophyll-a-Gehalt. Die Wirkung der Abwassernährstoffe ist im eutrophen Lake Shagawa in einem Versuch etwas grösser, im zweiten erheblich kleiner als der einzelnen oder in Kombination zugeführten reinen Nährstoffe. Im nährstoffarmen Wasser des Burntside River (= Zufluss) war sie etwa gleich gross wie die entsprechende Kombination reiner Nährstoffe. In keinem einzigen Fall bewirkte das Abwasser eine wesentlich höhere Wachstumssteigerung als die in mehr oder weniger gleicher Menge zugeführten reinen Nährstoffe.

Hatten diese Experimente nur die Wirkung reiner Nährstoffe und zweistufig gereinigten Abwassers erfasst, so erweiterten MILLER und MALONEY [6] in einer ähnlichen Versuchsanordnung diese Tests zur vollständigen Prüfung der reinen Nährstoffe und von zwei- und dreistufig gereinigtem Abwasser. Die Versuche wurden wie bei Malueg et al. [4] und Powers et al. [5] mit filtriertem Wasser aus dem Lake Shagawa und dem Burntside River und mit filtriertem Abwasser durchgeführt. Als Testobjekt diente Kulturmaterial von Selenastrum capricornutum. Über die P-Konzentration der geprüften Abwässer fehlen nähere Angaben. Da MALUEG et al. [4] und Powers et al. [5] das Abwasser aus der gleichen Modellkläranlage bezogen hatten, darf aber mit den gleichen Konzentrationen gerechnet werden, nämlich:

für zweistufig gereinigtes Abwasser etwa $5 \mathrm{mg} / 1 \mathrm{PO}_{4}-\mathrm{P}$ und

für dreistufig gereinigtes Abwasser etwa $50 \mu \mathrm{g} / 1 \mathrm{PO}_{4}-\mathrm{P}$.

Die Resultate sagen im wesentlichen folgendes aus:

- Mit einer Ausnahme (Phosphat im Lake-Shagawa-Wasser, Juli) fördern weder Nitrat noch Karbonat noch Phosphat (jeweils allein zugesetzt) das Algenwachstum.

- Düngung mit $\mathrm{P}+\mathrm{N}$ in Reinsubstanz fördert das Wachstum, desgleichen Düngung mit zweistufigem Abwasser.

- Dreistufig gereinigtes Abwasser wirkt nicht wachstumsfördernd. Phosphatzusatz hat düngende Wirkung.

Leider lassen diese Versuche keinen quantitativen Vergleich zu, da die Konzentrationen der reinen Nährstoffzusätze nicht mit jenen des Abwassers übereinstimmten. Aus der ganzen Versuchsserie vom Lake Shagawa ist aber an bemerkenswerten Einzelresultaten hervorzuheben:

5-10 Vol.-\% zweistufig gereinigtes Abwasser stimulieren die Phytoplanktonentwicklung sowohl eines oligotrophen als auch eines eutrophen Sees $[4,6]$, desgleichen in einer Verdünnung von $2 \%$ [5]. Entsprechende Düngung mit reinen $\mathrm{N}$ - und P-Salzen sowie deren Kombination mit Karbonat führt ungefähr zum gleichen Resultat. 5-10 Vol.-\% eines dreistufig gereinigten Abwassers mit einem Rest-P-Gehalt von $0,05 \mathrm{mg} / 1 \mathrm{P}$ blieb ohne Effekt $[4,6]$. Es ist hier zu bemerken, dass diese P-Konzentration höchstens $10 \%$ derjenigen im Abfluss heutiger europäischer dreistufiger Kläranlagen beträgt; mithin muss die Verdünnung, auf den P-Gehalt bezogen, auf rund $0,5 \mathrm{Vol} .-\%$ angesetzt werden. Eine solche finale Verdünnung von eingeleitetem Abwasser in einem See dürfte durchaus real sein. Im Einmündungsgebiet von abwasserbelasteten Vorflutern kann jedoch die Abwasser- bzw. die P-Konzentration höher sein und dadurch lokale Eutrophierungserscheinungen bewirken [1]. 
Wegen Streuungen der Einzelversuche sind diese Experimente wenig geeignet, Aussagen über die Ursachen von teilweise aufgetretenen Unterschieden zwischen abwassergedüngten und mit reinen Salzen gedüngten Grosskulturen zu machen. Es scheinen Faktoren mitzuspielen, die ausserhalb der Versuchskontrolle liegen, wenn man nicht mit statistischer Versuchsanordnung arbeitet. Bei den angewandten Dimensionen scheitert diese aber meist am erforderlichen Aufwand.

In die gleiche Richtung weisen die Resultate einer Arbeit von Shapiro und Ribeiro [7]. Wachstumstests mit Grünalgen (Scenedesmus sp.) und Blaualgen (Nostoc paludosum), die mit zweistufig gereinigtem Abwasser, mit reinem Phosphat und Nitrat und weiteren Salzen sowie mit zusätzlich behandeltem (chloriertem) Abwasser gedüngt worden waren, ergaben:

Im nährstoffreichen Wasser des Potomac River reagieren Blau- und Grünalgen auf Abwasserzugabe mit einem wesentlichen Biomasseanstieg. Reine Phosphatdüngung wird von den Grünalgen nur schwach beantwortet, da, wie Detailversuche zeigten, Nitratstickstoff das Wachstum limitierte, während die N-fixierende Nostoc allein durch Phosphat limitiert war. Zusätze von Ca, Mg, Silikat, Eisenzitrat und Spurenelementen blieben ohne Wirkung. Düngung mit zweistufig gereinigtem Abwasser, dem 98\% des ursprünglichen Phosphates entzogen worden waren, wurde von Grün- und Blaualgen mit minimalem Wachstum beantwortet. Düngung mit N-reduziertem Abwasser (Entzug von 62\% des aus Nitrat ammonifizierten Ammoniumstickstoffs) bewirkte bei den Grünalgen eine Verminderung des Wachstums auf $55 \%$ des ursprünglichen, durch zweistufig gereinigtes Abwasser bewirkten Wachstums $(=100 \%)$. Die Blaualgen zeigten dagegen eine Wachstumsrate von über $100 \%$. Die Autoren vermuten eine Wachstumshemmung durch den hohen Ammoniumgehalt des Abwassers.

Es ist anzumerken, dass diese Versuche mit Abwasser- bzw. Düngstoffkonzentrationen ausgeführt wurden, welche im praktischen Fall wohl immer sehr stark unterschritten werden. Im untersuchten Konzentrationsbereich zeigt das zweistufig gereinigte Abwasser eine Düngewirkung, die um so grösser ist, je geringer die Verdünnung in der Kultur ist. Es ist aber nicht ersichtlich, welcher Funktion diese Abhängigkeit folgt. Für die N-autonome Blaualge Nostoc paludosum darf angenommen werden, dass sie auf gleiche Zusätze von reinem und Abwasserphosphat gleich stark anspricht.

Eher in realitätsnahen Bereichen bewegen sich halbquantitative Experimente von Vallentyne et al. [8] über die Düngewirkung von Abwasser bzw. Phosphor auf das Phytoplankton des Erie- und des Ontariosees. Die Phosphorzugabe erfolgte hier in Form von zwei- und dreistufig gereinigtem Abwasser, dessen Phosphorgehalt mit reinem Phosphat auf die Konzentration des zweistufigen Abwassers aufgestockt worden war. Entsprechend dem populären Charakter der Arbeit fehlen quantitative Angaben. Die gewählte Testmethode, nämlich Membranfiltration einer gleichbleibenden Menge von Kultursuspension und visueller Vergleich der Filter, führt aber doch zu bemerkenswerten Feststellungen:

Gegenüber der Kontrolle fördert das dreistufig gereinigte Abwasser die Algenentwicklung nicht. Rohes, zweistufiges und mit P aufgestocktes dreistufiges Abwasser führt in der gleichen Versuchszeit $z u$ deutlich höheren, untereinander ungefähr gleich 
grossen Algendichten. Somit unterscheiden sich zwei- und dreistufig gereinigte Abwässer in ihrer Wirkung auf das Phytoplankton im wesentlichen nur durch den unterschiedlichen Phosphorgehalt.

Die artenmässige Zusammensetzung des Planktons wird durch Abwasserdüngung nicht beeinflusst, sofern ähnliche Versuche aus dem marinen Bereich auf Süsswasseralgen übertragen werden dürfen [11].

Aus den bisher besprochenen Arbeiten können gemeinsame Schlüsse gezogen werden:

1. Das Wachstum des Phytoplanktons und mithin dessen Produktion wird zur Hauptsache durch die beiden Nährstoffe Stickstoff und Phosphor bestimmt bzw. begrenzt. Damit werden die Resultate von Experimenten mit reinen Düngerzusätzen bestätigt.

2. Phosphor + Stickstoff lösen quantitativ ungefähr dieselbe Wachstumssteigerung aus, egal, ob sie nun in Form von rohem, mechanisch oder chemisch-biologisch gereinigtem Abwasser oder als reine Substanzen beigefügt werden.

Andererseits wurde z. B. anhand von langjährigen Untersuchungen des Phytoplanktons des Lake Washington [9] bewiesen, dass ein Entzug dieser Nährstoffe die Biomassesynthese herabsetzt. Während der Jahre 1941-1963 wurde zweistufig gereinigtes Abwasser in den Lake Washington eingeleitet. Dies hatte in dem seit 1933 untersuchten See eine massive Erhöhung des Phosphorgehaltes zur Folge. Die prozentuale Zunahme der Stickstoff- und Kohlenstoffkonzentration war relativ dazu kleiner. Die Produktion und Biomasse der Algen stieg bedeutend an; im Jahre 1963 erreichten die Nährstoff- und Biomassewerte ihren Höhepunkt. Von diesem Jahr an wurden die gereinigten Abwässer sukzessive vom See ferngehalten, nämlich 1963 28\%, 1968 $100 \%$ des gereinigten Abwassers. Die Nährstoffkonzentrationen und Biomassewerte gingen allmählich zurück. 1967/68 trat eine sprunghafte Besserung ein: Wurden 1963 noch die höchsten Sommer-Chlorophyllgehalte und die höchsten Winter-Phosphatgehalte gemessen, so sanken diese Zahlen 1968 gemeinsam auf ungefähr 30\% der Höchstwerte ab. Auch der Nitrat- und $\mathrm{CO}_{2}$-Gehalt ging zurück, wenn auch weniger stark. Im Jahre 1965 wurde bei Düngexperimenten mit Flaschenkulturen in situ beobachtet, dass eine kombinierte Phosphat-Nitrat-Düngung die Produktion des Phytoplanktons (gemessen an Chlorophyll, Photosynthese und Trockengewicht) am stärksten steigerte. Nitrat oder Phosphat allein erzielten zwar zeitweise einen Effekt; dieser blieb jedoch meist kleiner als derjenige der kombinierten Düngung. Zeitweise blieb er sogar ganz aus. Andere Nährstoffe, z. B. Spurenelemente oder Glukose, beeinflussten das Algenwachstum nicht.

Nährstoffbilanzberechnungen sowie die Korrelationsanalyse der überwachten Komponenten ergaben, dass der Lake Washington durch den Phosphor des jahrzehntelang zugeführten zweistufig gereinigten Abwassers viel tiefgreifender beeinflusst worden sein musste als durch jede andere Komponente. Interessant war nun die rasche Reaktion des Sees auf die kanalisationstechnisch realisierte Fernhaltung der Abwässer. Zwar benötigte der See eine sehr lange Zeit, um den akkumulierten Phosphor aus seinem gesamten Nährstoffkreislauf auszuscheiden. Durch den Unterbruch in der aktuellen Zufuhr neuen, d.h. allochthonen Phosphors war aber auch die aktuelle Alimentierung der sommerlichen photischen Schicht unterbrochen, eine Erscheinung, welche gegenwärtig, als Folge der konsequent durchgeführten P-Elimination 
in den Kläranlagen der Uferregion, auch im Zürichsee festgestellt werden kann [10]. Die Sommer-Chlorophyll- und Winter-Phosphatwerte sanken im Lake Washington fast unmittelbar auf die Höhe von Messwerten des Jahres 1950 ab.

Die Düngwirkung künstlicher Nährmedien unterscheidet sich nicht von jener des Abwassers und beruht im wesentlichen auf dem Gehalt an P und N. Diese experimentelle Erfahrung wird ferner durch die Tatsache ergänzt, dass ein Rückgang der "Dauerfütterung» von Seen durch eingeleitete Abwässer sehr rasch durch einen Rückgang der Biomassesynthese beantwortet wird, ohne dass unbekannte Fremdstoffe (z. B. Restverunreinigungen aus der P-Fällung, organische Restverunreinigungen, Komplexbildner, Vitamine im gereinigten Abwasser) diesen Effekt kompensieren würden. Dies lässt bei vorsichtiger Beurteilung der Sachlage den grundsätzlichen Schluss zu, dass mit der P-Elimination aus den Siedlungsabwässern mit Hilfe der chemischen Fällung (dritte Reinigungsstufe) nicht nur ein technisch gangbarer Weg beschritten wird, sondern dass diese Methode den ökologischen Effekt, dessentwegen sie propagiert, heute sogar vorgeschrieben und nun in zunehmendem Masse auch realisiert wird, tatsächlich erbringt.

\section{Diskussion}

Für das Tatsachenmaterial der vorliegenden Studie standen nur relativ wenige Arbeiten mit teilweise sehr verschiedenen Zielsetzungen und verschiedenen Arbeitsund Auswertemethoden zur Verfügung. Um aber die wissenschaftlich zwar triviale, für die Praxis aber zentral wichtige Frage über den Einfluss von gereinigtem Abwasser auf das Phytoplankton in Seen noch ausserhalb des Einflusses allfälliger politischer Entscheidungen beurteilen zu können, verzichten wir auf eine nochmalige, gründlichere, dafür aber zeitraubende experimentelle Bearbeitung. Das vorliegende Material erlaubt es, zumindest die die Praxis interessierenden Fragen mit hinreichender Sicherheit zu beantworten.

Jede der erwähnten Arbeiten basiert auf den Verhältnissen eines oder zwei bestimmter Gewässer oder auf künstlichen Randbedingungen. Angesichts der Individualität jedes Gewässers als Ökosystem können Resultate, welche mit In-situ-Versuchen gewonnen werden, nur unter Vorbehalten verallgemeinert werden. Um andererseits Resultate von Labor-Kulturexperimenten auf Gewässer zu übertragen, fehlen uns heute die Erfahrungen noch weitgehend. Die Interpretation der hier diskutierten Versuchsresultate muss sich - trotz verschiedenen Versuchsbedingungen - auf beobachtete Gemeinsamkeiten und gleichartige Ergebnisse stützen. Soll eine so gewonnene Erkenntnis Allgemeingültigkeit beanspruchen, so müssen sich die Versuchsbedingungen innerhalb eines grossen, aber bekannten Streuungsbereichs bewegen, und die Anzahl der Versuche sollte möglichst gross sein. Beides ist hier nicht der Fall. Trotzdem versuchen wir, Aussagen von einer gewissen Allgemeingültigkeit herauszuheben.

Die Natur des Abwassers: Bei den häuslichen Abwässern unterscheiden sich mechanisch gereinigte Abwässer qualitativ nicht von biologisch gereinigten Abwässern. Die Gesamtkonzentration an suspendierten, gelösten Stoffen nimmt bei der zweiten Reinigungsstufe $a b$, die Konzentration an gelösten anorganischen Stoffen jedoch eher zu. Generell ist ein gereinigtes Siedlungsabwasser ein Substrat, in welchem sämt- 
liche für die Algen notwendigen Nährstoffe in direkt zugänglicher Form vorliegen oder durch Stoffwechseltätigkeit der Mikroorganismen (ebenfalls im Abwasser enthalten) zugänglich gemacht werden können.

Charakter der Gewässer in bezug auf Nährstoffe und Algenwachstum: In sämtlichen untersuchten Gewässern sind Phosphat und Nitrat, einzeln oder kombiniert, die Minimumsfaktoren, welche die Synthese der Algenbiomasse primär begrenzen. Mit einer kombinierten Phosphat-Nitrat-Düngung konnte sowohl im Labor als auch in situ die Algenbiomasse erhöht werden. Andere Nährstoffe spielen eine geringe oder keine Rolle. Diese Wirkung wurde in oligotrophen und eutrophen Seen bei einer Konzentrationserhöhung von 10 bis etwa $2500 \mu \mathrm{g} / 1 \mathrm{PO}_{4}-\mathrm{P}$ festgestellt. Es wäre zwar denkbar, dass in vereinzelten Gewässern weder Phosphor noch Stickstoff, sondern andere Faktoren limitierend wirken, neben kurzzeitig oder sporadisch limitierenden Faktoren, wie Licht oder $\mathrm{CO}_{2}$. Für den Grossteil der amerikanischen und europäischen Binnengewässer ist dies jedoch unwahrscheinlich.

Aus den vorstehend mitgeteilten Ergebnissen, insbesondere aus der Tatsache, dass dreistufig gereinigtes Abwasser mit $50 \mu \mathrm{g} / 1 \mathrm{P}, \mathrm{zu} 10 \mathrm{Vol} .-\%$ verdünnt, das Wachstum des Phytoplanktons kaum fördert, wohl aber, wenn es durch künstlichen Zusatz auf $200 \mu \mathrm{g} / \mathrm{l} \mathrm{P}$ aufgestockt wird, kann geschlossen werden, dass phosphorarmes Abwasser in einem Gewässer, in welchem Phosphor das Algenwachstum limitiert, dieses nicht sichtlich stimulieren wird. Andere Faktoren, welche grössenordnungsmässig eine mit $\mathrm{P}$ und $\mathrm{N}$ vergleichbare Wirkung besitzen, gibt es offenbar nicht. Hinterher betrachtet ist dieser Schluss wissenschaftlich trivial; er hätte auch auf Grund theoretischer Erwägungen gezogen werden können, dann allerdings ohne Beweisführung. Für die Praxis ist er aber bedeutsam. Eine analoge Schlussfolgerung gilt auch für das Nitrat in N-limitierten Gewässern.

Die letztere Frage nach weiteren Wirkungsfaktoren, welche im unübersichtlichen Stoffkomplex "Abwasser» jederzeit zu erwarten sind, wurde bis heute zwar ausserhalb einer ansehnlichen Streuung als negativ beantwortet. Eine genauere Abklärung steht indessen noch aus. Verantwortlich dafür sind wohl zur Hauptsache methodische Schwierigkeiten. Unsere eigenen Versuche (S. 190) ergeben, dass in der gelösten Phase des zwei- und des dreistufig gereinigten Abwassers neben Phosphat und Nitrat keine weiteren Stoffe enthalten sind, welche das Algenwachstum unmittelbar beeinflussen. Eine Extrapolation auf den See ist aber angesichts der verwendeten LaborBatch-Kulturmethode zweifelhaft; die Versuche mögen jedoch Anstoss für weitere Arbeiten geben. Die In-situ-Versuche von Powers et al. [5] in Grosskulturen lieferten zum Teil widersprüchliche Resultate. Trotz den erwähnten Unzulänglichkeiten ist diese Technik kaum zu umgehen, wenn die Experimente nicht nur physiologisch, sondern als Einblicke in das Ökosystem verstanden werden, d. h. wenn die Gesamtreaktion der Biozönose einschliesslich jener der Bakterien beobachtet werden soll, welche gerade auf Abwasserzufuhr sehr rasch ansprechen und aus organischem Material anorganische Algennährstoffe bereitstellen können.

Ferner ist zu beachten, dass im Abwasser stets organische Komplexbildner vorhanden sein können. Diese können z. B. Spurenelemente maskieren und, sofern diese Stoffe Nährstoffunktion haben, das Wachstum hemmen, oder sie können dieses fördern, indem sie die Konzentration toxischer Ionen unter den toxischen Bereich senken [3]. Obschon solche Mechanismen vermutlich immer vorliegen, bewegt sich ihre 
Wirkung aber weit ausserhalb des Wirkungsspektrums der Primärnährstoffe. Ausgedehnte Düngeversuche, welche mit Nitrilotriazetat (NTA) in Grosskulturen in situ ausgeführt wurden [3], gehen jedenfalls ganz in dieser Richtung. Nicht ausser acht zu lassen ist das Eisen, das in unserem Lande heute das meistverwendete Fällungsagens für die dritte Stufe der Abwasserreinigung ist. $\mathrm{Da}$ es bei $\mathrm{pH}>7$ Kationen bindet, hat es, wie die Experimente von BürGI [3] zeigten, eine ähnliche Wirkung wie organische Komplexbildner. Eine besondere Wirkung als Oligo- oder Spurenelement hat Eisen (als dreiwertiges Ion) aber infolge seiner geringen Löslichkeit nicht.

\section{Schlussfolgerungen}

1. Die Wirkung gereinigten Abwassers auf das Phytoplankton von Seen kann durch die vorliegenden Ergebnisse aus eigenen Versuchen und Literaturarbeiten pauschal beurteilt werden. Im wesentlichen sind allein die Gehalte an Phosphor und Stickstoff für das Algenwachstum verantwortlich; weitere Faktoren spielen eine untergeordnete Rolle. Die im dreistufig gereinigten Abwasser vorhandenen Restmengen von Eisen haben keine merkliche wachstumsstimulierende Wirkung. Dies bedeutet, dass die dreistufige Reinigung (mechanisch-biologische Reinigung + chemische Fällung mit Eisen-III-chlorid oder -sulfat) keine unmittelbaren negativen A uswirkungen auf das Phytoplankton bzw. auf das Ökosystem hat.

2. Die Frage nach unbekannten Faktoren, welche neben $\mathrm{P}$ und $\mathrm{N}$ das Algenwachstum und das Artenspektrum des Phytoplanktons beeinflussen könnten und deren Anwesenheit im komplexen Stoffgemisch "Abwasser» denkbar ist, ist noch ungelöst. Bei der heute gebräuchlichen Komposition der Siedlungsabwässer ist sie allerdings zweitrangig.

\section{Zusammenfassung}

Die Wirkung von primären Nährstoffen, Metallsalzen und organischen Stoffen auf das Phytoplankton der Seen ist einigermassen bekannt. Die Primärnährstoffe P und $\mathrm{N}$ sind die für das Algenwachstum mit grossem Abstand dominierenden Komponenten. Andere Stoffe können die Produktion beeinflussen, aber gegenüber $\mathrm{P}$ und $\mathrm{N}$ nur in einem untergeordneten Ausmass. Diese Kenntnisse beziehen sich meist auf reine Zusätze; die Wirkung der bedeutendsten allochthonen Stoffquellen, nämlich der Abwässer, ist sehr schlecht bekannt.

Anhand eigener Experimente und Literaturarbeiten (letztere zur Hauptsache aus den USA) wird die Rolle des Abwassers als Träger von Primärnährstoffen und weiteren Wirkungsfaktoren analysiert. Auch bei Düngung mit Abwasser liegt der grösste wachstumssteigernde Effekt bei $\mathrm{P}$ und $\mathrm{N}$. Dreistufig gereinigtes (P-eliminiertes) Abwasser wirkt so wenig trophierend wie eine gleich konzentrierte reine Nährlösung. Eine Wachstumsstimulation durch andere Abwasser-Inhaltsstoffe liegt offensichtlich nicht vor. Auch die Resteisengehalte aus der chemischen Fällungsstufe (dritte Stufe der Abwasserreinigung) fördern das Algenwachstum nicht merklich. Somit hat das Verfahren der dreistufigen Abwasserreinigung (mechanisch-biologische Reinigung + chemische Fällung mit $\mathrm{Fe}^{3+}$ ) keine unmittelbaren negativen Auswirkungen auf das Phytoplankton bzw. auf das Ökosystem. 


\section{SUMMARY}

The effects of primary nutrients, metal salts, and organic matter on the phytoplankton in lakes is fairly well known. The primary nutrients $\mathrm{P}$ and $\mathrm{N}$ are by far the most dominant components influencing the growth of algae. Other compounds may have an effect on production, but to a much lesser extent than either $\mathrm{P}$ or $\mathrm{N}$. These findings are usually based on the addition of pure compounds. However, very little is known about the effect of wastewater discharges, the most important source of allochthonous nutrients.

The role of sewage effluent as a carrier for primary nutrients and other trace stimulatory factors has been analyzed by means of laboratory experiments and literature evaluation. When sewage is added to lake water, the increase of the phytoplankton production depends mainly on the concentration of $\mathrm{P}$ and $\mathrm{N}$. After tertiary treatment (P-removal), sewage has the same weak trophic effect as a pure solution containing the same nutrient concentrations. Growth-stimulating effects of other constituents which may be present in sewage effluents apparently do not exist. In the same way, residual amounts of iron, originating from the tertiary treatment, do not stimulate substantially the growth of algae. Hence, the method of three-stage wastewater treatment (mechanical-biological treatment + chemical precipitation with $\mathrm{Fe}^{3+}$ ) has no immediate negative effect on the phytoplankton or the aquatic ecosystem.

\section{RÉSUMÉ}

Les effets de substances nutritives primaires, de sels de métaux lourds et de matières organiques sur le phytoplancton des lacs sont connus dans une certaine mesure. Les substances nutritives primaires $P$ et $N$ sont, de loin, les composants les plus importants pour la croissance des algues. D'autres matières peuvent bien influencer la production, mais ce n'est qu'en faibles mesures par rapport au $\mathrm{P}$ et $\mathrm{N}$. Ces notions se rapportent, en général, à des substances pures; l'effet des plus importantes sources de matières allochtones, c'est-à-dire des eaux usées, est très mal connu.

Le rôle des eaux usées, comme porteur de matières nutritives primaires et d'autres facteurs, a été étudié d'après nos propres expériences et celles trouvées dans la littérature (ces dernières, d'origine des Etats-Unis). C'est aussi dans le cas d'une fertilisation par des eaux usées, que les plus grands effets de l'augmentation de la croissance sont dûs au $\mathrm{P}$ et $\mathrm{N}$. Des eaux usées, épurées en trois phases (élimination $\mathrm{du} P$ ), sont aussi peu fertilisantes qu'un pur milieu liquide de culture de la même concentration. Il n'y a donc manifestement pas de stimulation de croissance dûe à d'autres matières contenues dans les eaux usées. Les taux de fer restant après la phase de précipitation chimique (troisième phase d'épuration), eux aussi, ne stimulent pas sensiblement la croissance des algues. Ainsi, le procédé d'élimination des matières polluantes au cours de trois phases d'épuration (épuration mécanique et biologique+précipitation chimique avec $\mathrm{Fe}^{3+}$ ) n'a pas d'effet négatif directe sur le phytoplancton, voire sur l'écosystème.

\section{LITERATURVERZEICHNIS}

[1] Gächter, R., Szabó, E., und MAREš, A., Die lokale Beeinflussung eines stehenden Gewässers durch eine punktförmige Abwasserbelastung; ein divehter Nachweis der wachstumsbegrenzenden Wirkung des Phosphors im Vierwaldstättersee, Schweiz. Z. Hydrol. 33, 66-72 (1971).

[2] Wuhrmann, K., Some Problems and Perspectives in Applied Limnology, Mitt. Int. Ver. Limnol. $20,324-402(1974)$

[3] BürGI, H. R., Die Wirkung von NTA auf das Wachstum des Phytoplanktons, unter besondever Berücksichtigung des Eisens als Mikroelement, Schweiz. Z. Hydrol. 36/1, im Druck (1974).

[4] Malueg, K. W., Brice, R. M., Schults, D. W., und Larsen, D. P., The Shagawa Lake Project: Lake Restoration by Nutrient Removal from Wastewater Effluent, U. S. Environm. Prot. Agency, Ecol. Ser. Rep. EPA-R3-73-026 (1973).

5] Powers, C. F., Schults, D. W., Malueg, K. W., Brice, R. M., und Schuldt, M. D., Algal Responses to Nutrient Additions in Natural Waters. II. Field Experiments, Nutrients and Eutrophication, ASLO Spec. Symp. 1, 1.41-156 (1972). 
[6] Miller, W.E., und Malonex, T.E., Effects of Secondary and Tertiary Wastewater Effluents on Algal Growth in a Lake-River System, J. Wat. Poll. Contr. Fed. 43, 2361-2365 (1971).

[7] Shapiro, J., und Ribeiro, R., Algal Growth and Sewage Effluent in the Potomac Estuary, J. Wat. Poll. Contr. Fed. 37, 1034-1043 (1965).

[8] Vallentyne, J. R., Johnson, W. E., und Harris, A. J., A Visual Demonstration of the Beneficial Effects of Sewage Treatment for Phosphate Removal on Particulate Matter Production in Waters of Lakes Erie and Ontario, J. Fish. Res. Bd. Canada 27, 1493-1496 (1970).

[9] Edmondson, W. T., Nutrients and Phytoplankton in Lake Washington, Nutrients and Eutrophication, ASLO Spec. Symp. 1, 172-193 (1972).

[10] Тномаs, E. A., Oligotrophierung des Ziuvichsees, Vjschr. Naturf. Ges. Zürich 116, 165-179 (1971).

[11] Dunstan, W. M., und Menzel, D. W., Continuous Cultures of Natural Populations of Phytoplankton in Dilute, Treated Sewage Effluent, Limnol. Oceanogr. 16, 623-632 (1971). 